\section{LA REDACTION: CERTAINS CARACTERES DE METIER POUR PROFESSEURS PERPLEXES ET REDACTEURS DEBUTANTS}

\section{S. Major-Hamza \\ Rédactrice}

II y a quelques années, juste avant le début de la récession, fort d'une culture générale colorée mals surtout fière de quelques petits contrats de rédaction, j'al acquis pignon sur rue. En ce temps-1i, personne ne s'affichait comme rédacteur. Aufourd'hul on hésite encore à le falre, mals blentôt, cette profession méritera ses lettres de noblesse grâce à une marée montante de jeunes rédacteurs diplômés.

Evidemment, les rédacteurs d'expérlence par opposittion aux rédacteurs diplômés, ne courent pas les rues et sont en général, spécialisés. Listés comme rédacteur publicitalre, légal, scientifique, technique ou comme journaliste, éditorlaliste, scénariste, scripteur, auteur, $11 s$ n'ont guère d'intérêt pour les tâches de 1 'écrivain publique moderne quí prolifère sous le nom de rédacteur.

Ce nouveau-né a certes provoqué beaucoup de confusion. Pourtant, 11 suffit de penser à la hiérarchle médicale pour que sa place et ses tâches soient claires. Le rédacteur détient le même statut dans sa profession que le docteur en médecine générale. Tous deux s'occupent de l'ensemble de leur objet d'étude en dehors de toute spécialisation. Le scénariste, le journaliste, le rédacteur scfentifique sont à l'un ce que le pédiatre, le neurologue, le chirurgien sont à l'autre.

Un rédacteur est un professionnel qui êcrit des textes dans un délal donné, d'une certaine manière, sous une forme définitive et selon une formule prescrite. Cette définition s'applique à tous ceux qui vivent de leur plume. Ils sont appelés rédacteurs parce que leur objet d' étude n'est pas la langue ma1s le message. Ainst, le monde de la rédaction a une place précise pour chacun de ses spéclalistes même pour le littêra1re.
Il a fallu peu de temps pour que la profession se hlérarchise et entame une défintition de tâches. Depuis, mon pignon s'est écroulé avec la récession et je prépare maintenant une maftrise en rédaction.

Il est étonnant de constater combien de pratiques en rédaction professionnelle, professeurs et étudiants 1gnorent. D'aflleurs, ils ne posent que peu ou pas de questions sauf pour s'enquérir prudemment du genre de textes qu'un rédacteur est appelé à écrire. Le rédacteur est nouveau-né mais 11 a déjà une expérlence certalne à transmettre.

\section{TOUT CE QUI EST IMPRIME A ETE REDIGE PAR QUELQU'UN}

Les livres, la publicité sous toutes ses formes, les plèces de théâtre, les émissions de têlévision et de radio, les films, les cartes de souhaits, les revues, les journaux, les affiches, les lettres, les discours, les avis, les rapports sont tous des travaux qui touchent le rédacteur.

Le jeune rédacteur n'a pas à s'inquiéter de ce qu' 11 fera après ses études. Il n'a qu'à décider s'll veut se spéclaliser ou non. Il dolt, dans le premier cas, entrer en compétition avec les rédacteurs établis et, dans le deuxième cas, il dolt comprendre tous les mécantsmes de la sollicitation car le travall à la pige est, pour 1' Instant, la seule alternative. L'une ou l'autre de ces options ne promet en aucun cas la vie facile.

La rédaction est une profession très jeune. Il faudra des années pour mettre le public en confiance, pour perfectionner les programmes académiques, pour installer des maisons de rédaction professionnelle. Il suffit de se rappeler que les travaux conflés à l'assistant ImagInatif, au collègue artıstique, à la secrétałre talentueuse ou au parent instrult sont maintenant le domaine du rédacteur. Il ne suffit plus de bien écrire; 11 faut aussi définir les publicsible, déterminer les niveaux de langue appropriés, trouver les connotations parfaltes, les images, les méthaphores et les mots justes. 


\section{LE REDACTEUR EST AUSSI CORRECTEUR, RECHERCHISTE, EDITEUR, CONCEPTEUR, TRADUCTEUR ET GRAPHISTE}

Chaque rédaction a sa part de correction, de traduction, de conception, de remantements, de graphisme et exige toujours certaines recherches. Rédlger demande beaucoup de travall.

Le client fait toufours ce qu'11 peut pour faclliter la tâche du rédacteur. Malgré tout, les données de départ ne sont jamais complètes, ne sont pas nécessairement pertinentes, ne sont pas toujours en frança1s et sont rarement bien organisées.

Gare au rédacteur s'll n'a pas remarqué le numéro de téléphone manquant ou la faute d'orthographe dans le nom du cllent; s'11 n'a pas trouvé pertinent de vérifier une information erronnée; s'11 n'a pas falt les recherches nécessaires pour découvrir 1'lmage parfaite, presque évidente, qui aurait dû se trouver dans le texte; s'11 n'a pas suggéré à $1^{\prime}$ imprimeur la disposition graphique qui complimente a la perfection son texte.

Parmi toutes les qualités qu'un rédacteur se dolt de posséder, la méticulosité est vitale. Rien ne doft lứ échapper car 11 risque $d^{\prime}$ endommager sa réputation et de perdre ses cllents.

Lorsqu'un rêdacteur décroche un contrat, 11 doit souvent rédiger à part1r de peu ou de trop de données. Il n'est pas rare qu'un client approche un rédacteur sachant $q u^{\prime} 11$ a besoin d'un texte sans pouvoir en définir la nature, ou encore, qu' 11 impose au rédacteur beaucoup trop d'informations, les croyant toutes importantes. Le rédacteur dolt, dans les deux cas, falre des consultations, interviewer le client pour définir ses besolns, questionner ses collègues, visiter son lieu de travail pour finalement falre toutes les recherches connexes qui s'imposent.

Le jeune rédacteur ne maîtrise évidemment pas toutes les tâches quí ont traft a la rédaction. Il peut certes, les cultiver en acceptant des contrats parallèles. C'est d'allleurs une bonne façon de garantir sa survie.
Il peut travalller pour des individus (écrire une lettre, rédiger un curriculum vitae, corriger un texte), pour une compagnie (organiser la publication d'un bulletin mensuel, écrire des discours, rédiger des avis) ou encore pour une organtsation (rédiger un article, présenter un conférencler, volr aux textes de relations publiques).

11 peut aussi fatre du "ghostwriting" c'est à dife rédiger des articles, des nouvelles et même des livres qu'une autre personne aura le privilège de signer. C'est un domalne intéressant. Le rédacteur n'a drolt à aucune reconnalssance mals 11 est très bien payé.

\section{LA PART DE METIER DANS LA REDUCTION D'UN TEXTE, PAR UN PROFESSIONNEL}

Tous les rêdacteurs se souvlennent d'avoir fait à un moment ou à un autre, une bêtise majeure qu'lls ont payé cher. La part de métier dans la profession s'enselgne diffictlement mats 11 est certes possible de signaler certains détails importants.

Au départ, un rêdacteur se dolt le monter un "portfollo" c'est à dire un dossler de textes qu'11 a rédigés. C'est un outil de travall fort utile qui a l'avantage de dissiper les doutes que cllents et employeurs éventuels pourraient avoir quant à la compétence du rédacteur.

I1 importe également de comprendre que le rédacteur, même s'il maftrise sa langue, n'est pas dans le secret des dleux. Il est capable d'erreurs même s'll est extrêmement vigilant. C'est pourquol les rédacteurs professionnels engagent toujours des correcteurs d'épreuve et en fin de compte se dégagent de toute responsabilití. Personne n'est mort à cause d'une faute d'orthographe. Au p1s aller, la réputation du rédacteur subit quelques dommages.

Il faut faire attention au client qui trouve plaisir à prendre le rédacteur en défaut et 11 est préférable de refuser ses contrats. Il est la bête nolre du rédacteur. 
Le système des trois épreuves est un out11 que 1 'expérlence a prouvé efficace et très utile. Après une première consultation avec le client, lorsqu'un contrat est accepté, le rédacteur prépare une première épreuve. Ce document est une ébauche où les fautes d'orthographe ne sont pas corrigées, où les paragraphes ne sont pas définitifs, où le style n'est pas pol1. Il reflète entre autres l'idée générale, les concepts importants et le niveau de langue. Lorsqu'elle est prête, cette première épreuve dolt être Initialée par le client ce quí 1' empêche de changer d'idée et permet au rédacteur de poursuivre ses travaux.

Une deuxième épreuve est ensulte préparée. Ce document plus défintitif dolt également être inftialé. Si tout est accepté, le rédacteur prépare la dernière épreuve qu'll falt reviser par deux ou trols correcteurs. Lorsque le cllent accepte cette dernière épreuve, 11 est consclent que le rédacteur se dégage de toute responsabilité. Le rédacteur dolt à cet effet, falre imprimer une note sur ses factures. De plus, 11 a été remarqué que sl le rédacteur Imprime sur les feuilles des deux premiers documents, le mot épreuve, beaucoup de discussions inutiles sont évitées.

Les tarifs varient énormément. En gênéral, le rêdacteur est rémunéré selon un taux flxe à l'heure pour tous les travaux de consultation et de recherche. Les travaux de rédaction, traduction, correction se paient au mot c'est à dire aux cinq frappes. Il est difficile d' établir des tarifs. Plusieurs s'informent auprès des traducteurs de la communauté où 11 s veulent exercer leur profession pour s'enquérir de leurs tarifs. Alns1, 11 seuvent déterminer les leurs en étant sûrs d'être compétitifs.

\section{UN BON REDACTEUR DOIT LIRE, LIRE, LIRE, LIRE ET LIRE ENCORE}

Le rédacteur ne peut pas se permettre de faire preuve de snobisme. 11 est appelé à rédiger tous les genres de textes à des niveaux de langue très différents, dans des styles parfois originaux. C'est ce que 1a profession of fre de plus passionnant.
Le rédacteur se dolt d'acquérir un ensemble de connaissances qui lui permettent de développer le sens critique, le goût et le jugement. Il doit, pour se falre, 11re tout ce qui lui tombe sous 1a ma1n, garder les yeux ouverts et l'orellle attentive. Aucune information n'est négligeable. Bien écrire ne suffit pas.

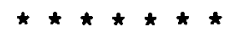

Suzanne Major-Hamza a trava11lé à Montréal pendant quelques années comme rédactrice. Actuellement elle falt des études de maftrise à 1'Université de Sherbrooke. 